\title{
Multistage artificial immune system for static VAR compensator planning
}

\author{
S. C. Mohd Nasir ${ }^{1}$, M. H. Mansor ${ }^{2}$, I. Musirin ${ }^{3}$, M. M. Othman ${ }^{4}$, T. M. Kuan ${ }^{5}$, K. Kamil ${ }^{6}$, \\ M. N. Abdullah ${ }^{7}$ \\ ${ }^{1,2,5,6}$ Department of Electrical Power Engineering, College of Engineering, Universiti Tenaga Nasional, Selangor, \\ Malaysia \\ ${ }^{3,4}$ Faculty of Electrical Engineering, Universiti Teknologi MARA, Selangor, Malaysia \\ ${ }^{7}$ Faculty of Electrical and Electronic Engineering, Universiti Tun Hussein Onn, Johor, Malaysia
}

\section{Article Info}

Article history:

Received Jun 7, 2018

Revised Oct 10, 2018

Accepted Nov 25, 2018

Keywords:

Multistage artificial immune system

Static VAR compensator

Total system loss minimization

Voltage profile improvement

\begin{abstract}
Interconnected network of transmission and distribution lines lead to losses in the system and weakening the voltage stability in the system. Installing Static VAR Compensator (SVC) in power system has known to improve the system by minimizing the total loss and improve the voltage profile of the system. This paper presents the application of Multistage Artificial Immune System (MAIS) technique to determine optimal size of SVC. The performance of this technique is tested on the IEEE 14-Bus Reliability Test System (RTS). The optimization results show that the proposed Multistage Artificial Immune System (MAIS) technique gives better solution of SVC compensator planning problem compared to single stage Artificial Immune System (AIS) in terms of lower total system loss and improved minimum voltage magnitude.
\end{abstract}

Copyright (C) 2019 Institute of Advanced Engineering and Science. All rights reserved.

\section{Corresponding Author:}

M. H. Mansor,

Department of Electrical Power Engineering, College of Engineering,

Universiti Tenaga Nasional 43000 Kajang, Selangor, Malaysia.

Email: mhelmi@uniten.edu.my

\section{INTRODUCTION}

Electrical power systems are complex systems consisting of large number of generating units and interconnected network of transmission and distribution lines. Furthermore, the power demand is increasing at an alarming rate day by day, as a result of which the utilities are being compelled to operate their generating units and transmission lines at their maximum capacity [1]. In order to improve the power system operation, Flexible AC Transmission Systems (FACTS) devices are used in recent years. Reactive power changes produced by load variations and line switching can cause adverse effects on system voltage stability and the interconnected system security [2].

There are several types of FACTS devices such as Static Synchronous Compensator (STATCOM), Static VAR Compensator (SVC), Unified Power Flow Controller (UPFC), Convertible Series Compensator (CSC), Inter-phase Power Flow Controller (IPFC), Static Synchronous Series Controller (SSSC), Thyristor Controlled Series Compensator (TCSC), Thyristor Controlled Phase Shifting Transformer (TCPST) and Super Conducting Magnetic Energy Storage (SMES) [3]. Among the types of FACTS devices, SVC is the prominently used by the Grid System Operators (GSOs). One of the reasons for this is SVC is cheaper than other FACTS devices especially STATCOM and TCSC. However, SVC is still very expensive in price thus its optimal location and size in the network must be optimally ascertained [3].

For the past decade, researchers and electrical engineers have introduced many optimization techniques for the SVC planning. These techniques include mathematical methods like linear programming, quadratic programming and heuristic methods like Evolutionary Programming (EP), Particle Swarm 
Optimization (PSO) [4] and Artificial Immune System (AIS). AIS is a computational paradigm that belong to the computational intelligence family inspired by the biological immune system [5]. The AIS uses an idea gleaned from immunology in order to develop systems capable of performing different tasks in various areas of research [6]. AIS is a diverse area of research that attempts to bridge immunology and engineering developed through the application of techniques such as mathematical and computational modeling of immunology. AIS has proven capable of solving many power system problems such as economic dispatch problem, distributed generation placement problem and reactive power planning.

This paper proposes to use Multistage Artificial Immune System (MAIS) technique to solve SVC planning problem. MAIS is a new technique that developed from the modification of the original AIS technique. Different from the original AIS, MAIS has multiple stages of the same processes of cloning, mutation and selection. The purpose of this modification is to make the possible solution broader than the original AIS technique. Subsequently, this will increase the accuracy of the results produced as the number of comparison increased. The implementation of MAIS to solve SVC planning problem is explained in details in the next section.

It is found that the proposed MAIS is superior than the original AIS to solve SVC planning problem. MAIS gives better solution in terms of lower total system loss and improved minimum voltage magnitude compared to original AIS.

\section{PROBLEM FORMULATION}

Prior to the employment of MAIS, the optimal location of the SVC units was found using voltage deviation index. Voltage deviation index (VDI) is an indicator used to determine how much the bus voltage magnitude has deviated from its reference value. A power system is considered to have good voltage profile if it has low voltage deviation index value. The formula of voltage deviation index is as shown in equation (1).

$$
\Delta V_{\Sigma}=\sum_{i=1}^{b}\left(\frac{V_{\text {ref }, i}-V_{i}}{V_{\text {ref }, i}}\right)^{2}
$$

$$
\text { where } V_{\text {ref,i }} \text { is the reference voltage at } i^{\text {th }} \text { bus [8] }
$$

The search for the optimal location and size is based on two objective functions, which are to minimize total system loss and to improve minimum voltage magnitude. This study considers single objective optimization, which means only one objective function is optimized at a time.

\section{a. Objective Function 1: To minimize total system loss}

The total system loss can be calculated using the following equation:

$$
P_{\text {loss }}=\sum_{l=1}^{b r} R_{l} I_{l}^{2}=\sum_{i=1}^{b} \sum_{\substack{j=1 \\ i \neq j}}^{b}\left[V_{i}^{2}+V_{j}^{2}-2 V_{i} V_{j} \cos \left(\delta_{i}-\delta_{j}\right)\right] Y_{i i} \cos \varphi_{i j}
$$

where $b r$ and $b$ are the number of lines and buses, respectively; $R_{l}$ is the resistance of line $l^{\text {th }} ; I_{l}$ is the current through line $l^{\text {th }} ; V_{i}$ and $\delta_{i}$ are the voltage magnitude and angle at node $i^{\text {th }}$, respectively; $Y_{i j}$ and $\varphi_{i j}$ are the magnitude and angle of the line admittance between bus $i^{t h}$ and bus $j^{t h}$, respectively [8].

\section{b. Objective Function 2: To improve minimum voltage magnitude \\ $V_{\min (\text { after } S V C)} \geq V_{\min (\text { before } S V C)}$}

For this second objective function, it is ensured that the minimum voltage magnitude of the system is improved after the installation of SVCs in the power system as shown in equation (3).

\section{c. Operational Constraints}

The two objective functions are subjected to the following operational constraints:

\subsubsection{Real and Reactive Power Balance Contraints}


The balance of active and reactive powers must be satisfied at each bus. Real and reactive power balance with respect to a bus can be formulated as:

$$
\begin{aligned}
& P_{G i}-P_{L i}=V_{i} \sum_{j=1}^{n}\left[V_{j}\left[G_{i j}^{\prime} \cos \left(\delta_{i}-\delta_{j}\right)+B_{i j}^{\prime} \sin \left(\delta_{i}-\delta_{j}\right)\right]\right] \\
& Q_{G i}-Q_{L i}=V_{i} \sum_{j=1}^{n}\left[V_{j}\left[G^{\prime}{ }_{i j} \sin \left(\delta_{i}-\delta_{j}\right)-G^{\prime}{ }_{i j} \sin \left(\delta_{i}-\delta_{j}\right)\right]\right]
\end{aligned}
$$

where $P_{G i}$ and $Q_{G i}$ are the generated active and reactive powers, respectively; $P_{L i}$ and $Q_{L i}$ are the load active and reactive powers at node $i$, respectively. The conductance, $G_{i k}^{\prime}$ and susceptance, $B^{\prime}{ }_{i k}$ represent the real and imaginary components of element $Y^{\prime}{ }_{i j}$ of the $\left[Y^{\prime}{ }_{b b}\right]$ matrix, respectively, obtained by modifying the initial nodal admittances matrix when introducing the SVC [10].

\subsubsection{Voltage Stability Constraint}

The voltage magnitude at all busses are ensured to be within the limits of $\pm 5 \%$ of its nominal value.

$$
\mathrm{V}_{\mathrm{i}, \min } \leq \mathrm{V}_{\mathrm{i}, \mathrm{nom}} \leq \mathrm{V}_{\mathrm{i}, \max }
$$

\section{IMPLEMENTATION OF MAIS TO FIND THE SIZE OF SVCS}

The location of SVC units are determined by using voltage deviation index before the MAIS is employed to find the optimal size of the SVC units. For the IEEE 14-Bus Reliability Test System (RTS) as shown in Figure 1, it was decided to install only three SVC units based on its size. The locations of the SVC units are at buses that have highest VDI values.

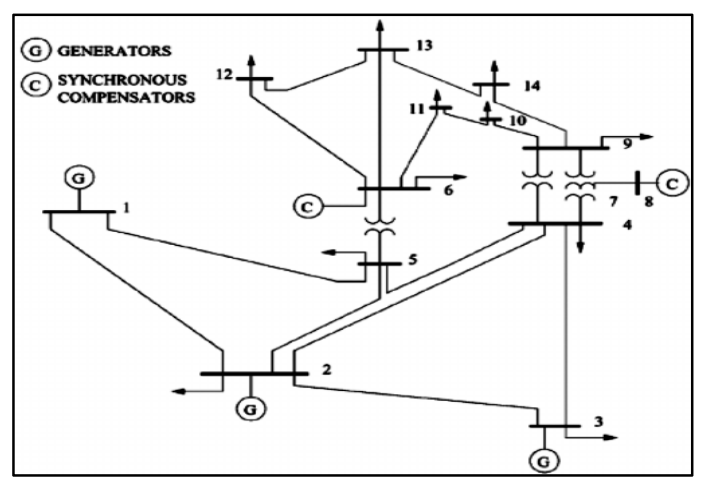

Figure 1. 14-Bus Reliability Test System [9]

Table 1. Voltage Deviation Index at Base Condition

\begin{tabular}{cc}
\hline Bus & $\begin{array}{c}\text { Voltage deviation } \\
\text { Index at each bus }\end{array}$ \\
\hline 1 & 0.0036 \\
2 & 0.0020 \\
3 & 0.0001 \\
4 & 0.0002 \\
5 & 0.0006 \\
6 & 0.0049 \\
7 & 0.0031 \\
8 & 0.0081 \\
9 & 0.0097 \\
10 & 0.0097 \\
11 & 0.0066 \\
12 & 0.0034 \\
13 & 0.0032 \\
14 & 0.0039 \\
\hline
\end{tabular}


The process of MAIS to find the optimal size of the three SVC units is shown in Figure 2. Firstly, three initial values were generated randomly. These three values represent three control variables of SVC units to be installed. The series of random number, $x_{i}$ is generated by uniform distribution number [10]:

$x_{i}=$ rand $(\mathrm{a}, \mathrm{b}) *$ off_set + min_set

where;

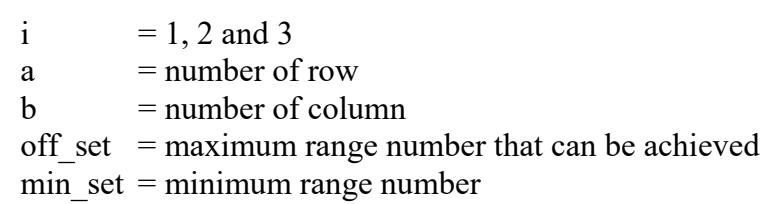

The random number generated using the uniform distribution number will be assigned as the size of the SVC units. Since it was decided to have three SVCs installed, thus three variables namely $x_{1}, x_{2}$ and $x_{3}$ are generated to represent the size of SVC at bus 8,9 and 10 respectively. The range of random number of the SVC units' size is between -100 MVAR to 100 MVAR. The SVC units can either injecting or absorbing the reactive power to or from the buses respectively.

Equations (3), (4), (5) and (6) are used to ensure the random numbers generated abide the problem constraints. Ttwenty individuals of control variables are generated based on the calculated fitness, which can be total system loss or minimum voltage magnitude. These individuals are stored in the population pool. Subsequently, the twenty individuals are cloned by ten.

Unlike the single stage AIS, MAIS clones the population with multiple stages. Before the cloning process, the initial population are decomposed into four sub-populations, which contains five individuals. After that, the sub-populations will undergo cloning, mutation and selection processes. The MATLAB programming code for cloning process is as shown in equation (8).

$$
\text { Clone_k }=\operatorname{repmat}(\mathrm{A},[\mathrm{i}, \mathrm{j}])
$$

where;

$$
\begin{array}{ll}
\mathrm{k} & =\text { number of stage } \\
\mathrm{A} & =\text { subject to clone } \\
\mathrm{i} & =\text { clone the row } \\
\mathrm{j} & =\text { clone the column }
\end{array}
$$

For the mutation process, Gaussian Mutation technique is used to produce the off spring. The Gaussian Mutation equation is as shown in equation (9).

$$
x_{i+m, j}=x_{i, j}+N\left(0, \beta\left(x_{j \max }-x_{j \min }\right)\left(\frac{f_{i}}{f_{\max }}\right)\right)
$$

where;

$$
\begin{array}{ll}
x_{i+m, j} & =\text { mutated parent (offspring) } \\
x_{i, j} & =\text { parents } \\
N & =\text { Gaussian random variable with mean } \mu \text { and variance } \gamma^{2} \\
\beta & =\text { mutation scale, } 0<\beta<1 \\
x_{j \text { max }} & =\text { maximum random number for every variable } \\
x_{j \text { min }} & =\text { minimum random number for every variable } \\
f_{i} & =\text { fitness for the } i^{t h} \text { random number } \\
f_{\text {max }} & =\text { maximum fitness }
\end{array}
$$




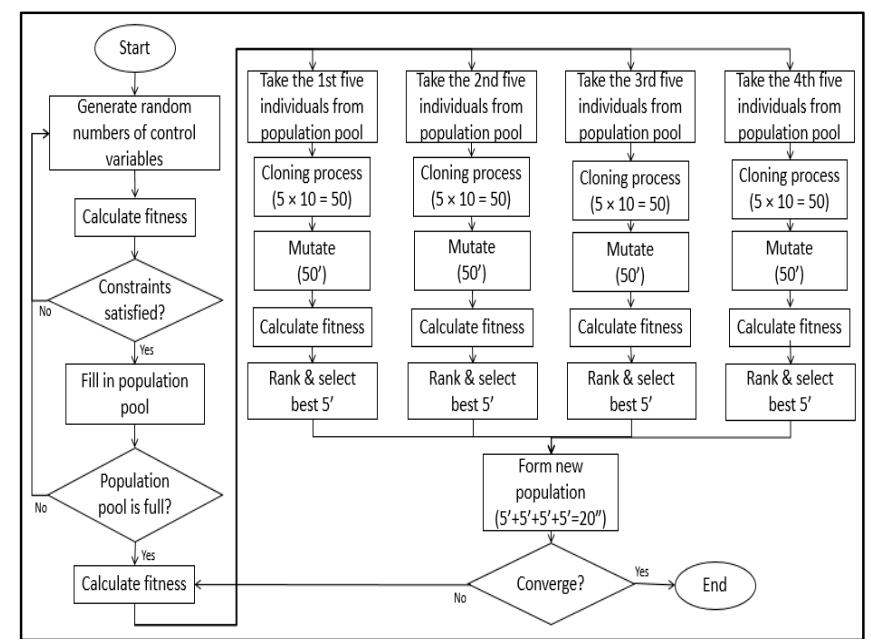

Figure 2. Flow Chart for MAIS Technique

At the end of each stage, five best individuals are produced made up of twenty individuals. The best twenty individuals are then undergo convergence test to find the global optima.

Convergence criterion is specified when the value of difference between the $20^{\text {th }}$ and the $1^{\text {st }}$ individual is less than 0.00001 for each fitness. Mathematically, the convergence criterion of fitness of total system loss and minimum bus voltage are as shown in equation (10) and equation (11) respectively.

$$
\begin{gathered}
P_{\text {loss }, \max }-P_{\text {loss }, \min } \leq 0.0001 \\
\Delta V_{\Sigma \max }-\Delta V_{\Sigma, \min } \leq 0.0001
\end{gathered}
$$

\section{RESULTS AND DISCUSSION}

As mentioned in the previous section, there are two objective functions tested for the MAIS optimization. The objective functions are to minimize total system loss and to improve minimum bus voltage. Therefore, the results of MAIS to determine the size of the installed SVC units are divided into two parts based on its objective functions. For these two objective functions, the locations of the SVC units are at buses 8,9 and 10. These locations are determined from the VDI values of all buses where the three SVC units are placed at the buses that have highest VDI values.

\section{a. Minimizing Total System Loss as the Objective Function}

The results of total system loss and minimum voltage magnitude produced before and after the optimization for the first objective function are tabulated in Table 2.

Table 2. Values of Total System Loss and Minimum Voltage Magnitude for Minimizing the Total System Loss

\begin{tabular}{ccc}
\hline Technique & $\begin{array}{c}\text { Total System } \\
\text { Loss (MW) }\end{array}$ & $\begin{array}{c}\text { Minimum } \\
\text { Voltage } \\
\text { Magnitude (p.u) }\end{array}$ \\
\hline $\begin{array}{c}\text { Pre-optimization (without SVC) } \\
\text { Post-optimization (using AIS } \\
\begin{array}{c}\text { technique) } \\
\text { Post-optimization (using MAIS } \\
\text { technique) }\end{array}\end{array}$ 13.56 $^{13.48}$ & 1.0100 \\
& 13.46 & 1.0100 \\
\hline
\end{tabular}

Based on the Table 2, MAIS produced the lowest total system loss compared to pre-optimization and AIS results, which is $13.46 \mathrm{MW}$. However, for this objective function, the minimum voltage magnitude produced by MAIS is slidely lower than pre-optimization and AIS results. This value is still acceptable as the most minimum value allowed is 0.95 p.u. It is found that SVC units installation can help to minimize the total 
system loss and by using MAIS technique to find the optimal size of the SVC units will lead to better results compared to AIS technique.

Table 3. Locations and Sizes of SVC Units Based on Objective Function of Minimizing the Total System Loss

\begin{tabular}{ccccc}
\hline & \multirow{3}{*}{ Technique } & \multicolumn{3}{c}{ SVC location } \\
\cline { 3 - 5 } Size of SVC & & Bus 8 & Bus 9 & Bus 10 \\
\cline { 2 - 5 } (MVAR) & AIS & 34.35 & 35.25 & 2.79 \\
& MAIS & -25.83 & 32.28 & 15.29 \\
\hline
\end{tabular}

It can be seen from Table 3. that all the three SVC units supplied reactive power to the system. However, for MAIS, only SVCs at bus 9 and bus 10 supplied reactive power and SVC at bus 8 absorbed reactive power of 25.83 MVAR from the system. Different technique leads to different size of SVC units.

Table 4. Values of Total System Loss and Minimum Voltage Magnitude for Improving the Minimum

\begin{tabular}{ccc}
\multicolumn{3}{c}{ Voltage Magnitude } \\
\hline Technique & $\begin{array}{c}\text { Total System } \\
\text { Loss (MW) }\end{array}$ & $\begin{array}{c}\text { Minimum Voltage } \\
\text { Magnitude (p.u) }\end{array}$ \\
\hline $\begin{array}{c}\text { Pre-optimization (without SVC) } \\
\text { Post-optimization (using AIS } \\
\text { technique) }\end{array}$ & 13.56 & 1.0100 \\
$\begin{array}{c}\text { Post-optimization (using MAIS } \\
\text { technique) }\end{array}$ & 13.49 & 1.0150 \\
\hline
\end{tabular}

Based on the results in Table 4. the minimum voltage magnitude of the system improved from 1.010 p.u before optimization to 1.015 p.u and 1.020 p.u after it is optimized using AIS and MAIS respectively. The total system loss also can be seen reduced from the pre-optimization. From Table 5. it can be seen that both AIS and MAIS have SVC units that absorbing the reactive power. For AIS, SVC unit at bus 8 absorbed the reactive power while for MAIS at bus 10. The other two SVC units of each technique are supplying reactive power to the system.

Table 5. Locations and Sizes of SVC Units Based on Objective Function of Improving the Minimum Voltage Magnitude

\begin{tabular}{ccccc}
\hline \multirow{2}{*}{ Size of SVC } & \multirow{2}{*}{ Technique } & \multicolumn{3}{c}{ SVC location } \\
\cline { 2 - 5 }$($ MVAR) & & Bus 8 & Bus 9 & Bus 10 \\
\cline { 2 - 5 } & AIS & -23.30 & 3.88 & 66.19 \\
& MAIS & 64.94 & 50.59 & -67.41 \\
\hline
\end{tabular}

\section{CONCLUSIONS}

In this paper, MAIS optimization technique has been used to achieve two different objective functions for determining the optimal size of three SVC units installed in the IEEE 14-Bus Reliability Test System (RTS). The locations of the SVC units were firstly determined using the VDI approach. When targeting one of objective function, the other objective function is slightly having disadvantage. However, focusing on each objective will help the Grid System Operators (GSOs) to compare and decide which objective need to be considered depending on the current situation and condition. It can be concluded that MAIS is superior than the original AIS for solving static VAR compensator planning problem in terms of giving lower total system loss and improving minimum voltage magnitude. This due to multiple stages of the same processes of cloning, mutation and selection in MAIS that increase the potential of produced individuals in initial population to be selected as the final optimal solution.

For the future development, the MAIS algorithm can be improved by replacing the Gaussian Mutation technique with Lognormal Mutation technique or mutated using other optimization techniques such as Particle Swarm Optimization (PSO) or Kinetic Gas Molecule optimization (KGMO) to produce better offspring from the mutation process.

\section{ACKNOWLEDGMENT}

Multistage artificial immune system for static VAR compensator planning (S. C. Mohd Nasir) 
The authors would like to acknowledge the Universiti Tenaga Nasional (UNITEN) for the financial support of this project. This research is supported by UNITEN under the UNITEN Internal Research Grant (UNIIG2018) with project code: J510050629.

\section{REFERENCES}

[1] S. R. Ola, "Reactive Power Flow Control Using Static VAR Compensator to Improve Voltage Stability in Transmission System," 2016.

[2] L. A. Snider, "An Intelligent Voltage Controller for Static VAR Compensators," pp. 239-243, 1994.

[3] A. K. Mohanty and A. K. Barik, "Power System Stability Improvement Using FACTS Devices," Int. J. Mod. Eng. Res., vol. 1, no. 2, pp. 666-672, 2009.

[4] M. Gitizadeh, M. Shidpilehvar, and M. Mardaneh, "A new method for SVC placement considering FSS limit and SVC investment cost," International Journal of Electrical Power and Energy Systems, vol. 53. pp. 900-908, 2013.

[5] J. Al-Enezi, M. Abbod, and S. Alsharhan, "Artificial Immune Systems-models, algorithms and applications," Int. J. Res. Rev. Appl. Sci., vol. 3, no. May, pp. 118-131, 2010.

[6] W. Ongsakul and P. Jirapong, "Optimal Allocation of FACTS Devices to Enhance Total Transfer Capability Using Evolutionary Programming," pp. 4175-4178, 2005.

[7] M. H. Mansor, M. R. Irving, and G. A. Taylor, "A decomposition/aggregation method for solving electrical power dispatch problems," in 2012 47th International Universities Power Engineering Conference (UPEC), 2012, pp. 17.

[8] K. P. Nguyen, G. Fujita, and V. N. Dieu, "CUCKOO SEARCH ALGORITHM FOR OPTIMAL PLACEMENT AND SIZING OF STATIC VAR COMPENSATOR IN LARGE-SCALE POWER SYSTEMS,” vol. 6, no. 2, pp. 59-68, 2016.

[9] D. R. Tobergte and S. Curtis, Transmission and Distribution Electrical Engineering 3rd Edition. Newnes, 2013.

[10] R. Sirjani, A. Mohamed, and H. Shareef, "A Hybrid BCO / HS Algorithm for Optimal Placement and Sizing of Static Var Compensators in Power Systems," pp. 54-59.

[11] R. Sirjani, A. Mohamed, and H. Shareef, "Optimal allocation of shunt Var compensators in power systems using a novel global harmony search algorithm,” Int. J. Electr. Power Energy Syst., vol. 43, no. 1, pp. 562-572, 2012.

Indonesian J Elec Eng \& Comp Sci, Vol. 14, No. 1, April 2019 : 346 - 352 